\title{
A novel fast-qualitative balance test method of screening for vestibular disorder patients
}

\author{
Tran Anh Vu', Hoang Quang Huy ${ }^{1}$, Le Van Tuan ${ }^{1}$, Pham Thi Viet Huong ${ }^{2}$ \\ ${ }^{1}$ School of Electronics and Telecommunications, Hanoi University of Science and Technology, Hanoi, Vietnam \\ ${ }^{2}$ International School, Vietnam National University, Hanoi, Vietnam
}

\section{Article Info \\ Article history: \\ Received Jun 29, 2021 \\ Revised Dec 8, 2021 \\ Accepted Dec 16, 2021}

Keywords:

Balance test method

Image processing

Marker-based techniques

Movement analysis

Vestibular disorder

\begin{abstract}
Body balance test is one of the methods of assessing vestibular level. However, the results are still qualitative, depending on the subjectivity of the doctor. This study proposes a new, low-cost method to quantitatively determine the degree of body imbalance. The proposal includes a low-cost laser source, a proposed rectangular paper frame, a camera, and a computer. The rectangular frame is mounted on the patient. The laser source is fixed and projected onto this rectangular frame. The laser projection point is taken as the origin point to evaluate the movement of the frame, which is also the movement of the patient's body. This rectangular frame is pre-marked with points to get more accuracy of the position of the laser point. Therefore, this measurement is not affected by the position of the camera during recording. The video is then procecced by computer to determine the position of laser point, it is also presented the movement of the patient's body. Initial trials were conducted on vestibular and normal patients. The results show that there is a clear difference in the balance of the vestibular and healthy people. The proposed method can be used to support quantitative screening for vestibular disease.
\end{abstract}

This is an open access article under the CC BY-SA license.

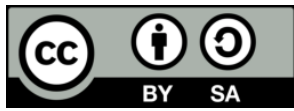

\section{Corresponding Author:}

Pham Thi Viet Huong

International School, Vietnam National University, Hanoi, Vietnam

144 Xuan Thuy, Cau Giay, Hanoi, Vietnam

Email: huongptv@isvnu.vn

\section{INTRODUCTION}

Vestibular disorders are becoming popular all around the world. According to this postural measure, about $35 \%$ of US individuals aged 40 and up exhibited signs of balance dysfunction, this ratio rises dramatically with age, reaching $85 \%$ of those aged 80 and up [1]-[3]. The vestibular disease has a significant impact on the patient's everyday's life [4]. Furthermore, long-term vestibular problems are linked to a variety of serious illnesses, including stroke, hypotension, and heart attack [5], [6]. As a result, there is an urgent need for the therapy, which is driven by the incidence and severity of vestibular diseases.

According to literature, the most well-known procedures for diagnosing vestibular problems are hearing tests, eye movement tests and balance tests [7]-[9]. In the hearing tests, we need earphones, electrodes, and a quiet room [7], [10]. Despite the fact that it produces precise findings, it takes time for doctor to inspect every single patient. Eye movement tests are another way to check for vestibular problems. If a patient displays significant anomalies in voluntary and reflexive eye movements, a referral to neurology or neuroophthalmology may be necessary [2]. A few examples of this approach can be Electronystagmography (ENG), Videonystagmography (VNG) [11], rotation tests [7], benign paroxysmal positional vertigo (BPPV) of the posterior semicircular canal [12], Electrovestibulography (EVestG) [13]. Another method requires averaging of muscle activity thus are termed vestibular evoked myogenic potentials 
(VEMPs) [14], [15]. Although these methods give accurate findings; but it is expensive and complicated, making it unsuitable for screening.

There are several method to determine human's body movement. In [16], video head impulse test is used in elderly individuals, hence can detect vestibular disorder in the elderly. Red, green, and blue (RGB) videos of 3D body models is used in assess general movement [17]. In children, the typical approach to estimate the balance deficits is timed one leg stance task [18]-[20], or based on the physical fitness outcomes [21], or based on model-based clustering [22], or based on cognitive process [23].

Vestibular disorders often make patients unable to keep their balance. When patients have vestibular dysfunction, visual information may be sufficient to eliminate imbalance and maintain stable posture. When the visual input is removed, this is the main idea of the Romberg test [24], [25]. Bodily instability due to lack of vision is easily exposed, especially when requiring the patient to stand in particular positions, which produce different information about the position of the joints [26]. Therefore, balance testing has long been considered one of the best methods for detecting vestibular disorders. In a balance test, the test is done for a short time to determine how well the body is able to balance. The Romberg test comes in two different versions: the original test and the tandem Romberg test. In the original Romberg test, the patient stands with his feet together and his arms crossed in front of his body. The patient tries to keep his balance with his eyes open and then closed. Vestibular level is assessed based on how long the patient can balance with eyes closed. The tandem Romberg test is a modification of the patient's standing position from the original Romberg test. In this test, the patient stands with both feet in a heel-to-toe position, one leg straight in front of the other, first with eyes open and then with eyes closed. The patient's arms are crossed in front of his chest and his open palm rests on the opposite shoulder. The findings of the Romberg test were refined to provide an objective assessment of postural stability [27], [28]. The Romberg test can be used as a rapid screening tool for vestibular disease in the clinic [29]. However, it still has significant disadvantages such as the inability to accurately detect the patient's sway [30], [31]. In particular, it does not create a database that records the patient's status quantitatively through the tests.

In this study, we proposed a method that have the ability to overcome the disadvantages of the previous approach that it does not depend on the observation and the objective of doctors. Our proposed method can evaluate the patient's balance quantitatively, which is more accurate and less time cosuming. In our method, we propose a low-cost and flexible computer-aided Romberg test assesment system that can assess the patient's balance through the Romberg test and store the data for future purposes. Our system includes a laser pointer, a small rectangular paper frame, a camera and a computer, which we will talk in detail in the next part. The system automatically provides quantitative results on people's balance disorder and this data can be kept for assessing the patient's ability to recover during treatment and later. Our paper has four section: section 1 is the introduction, section 2 provides our proposed method, section 3 evaluates the experiment, and section 4 concludes the paper.

\section{RESEARCH METHOD}

\subsection{Designed system}

The proposed system for use during the Romberg test is presented in Figure 1, for automated quantitative assessment of body balance. Components of the system include: a low-cost laser pointer, a small rectangular paper frame, a camera, and a personal computer for data processing. The process of performing a body balance assessment is performed:

- The paper frame is attached to the back of the patient's body.

- A red laser pointer is projected onto the frame and held steady during measurement. This laser point is used as a reference point for motion.

- The camera records the frame's movement relative to the reference laser point.

- The video data is then processed to determine the movement of the frame relative to the reference laser point, which is also the movement of the body.

As shown in Figure 1, the proposed frame is attached to the patient's back [32]. Thus, when the patient moves, frame also moves, the camera records this movement [33], [34]. This is known as Markerbased technique [35], [36], the frame in this study is considered as a marker, which is attached to the target region, such as limb, joint, or body part. And the position and orientation of the target region will be tracked. This technique is widely applied in sports, rehabilitation, and biomechanical [37]-[44].

In order to facilitate processing, the frame is designed with a white background and blue border. This makes the image easily identifiable in case the patient wears a shirt of the same color with the background. To calculate the movement of the laser point on the frame more accurately, on the white background of the frame 15 pairs of black dots are designed, each pair is $1 \mathrm{~cm}$ apart as shown in Figure 2. 


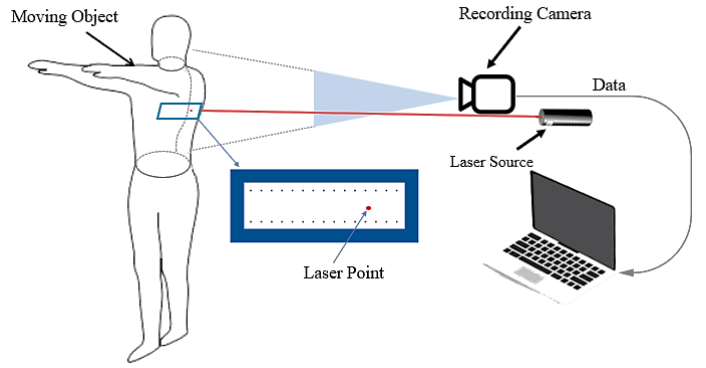

Figure 1. The proposed system components

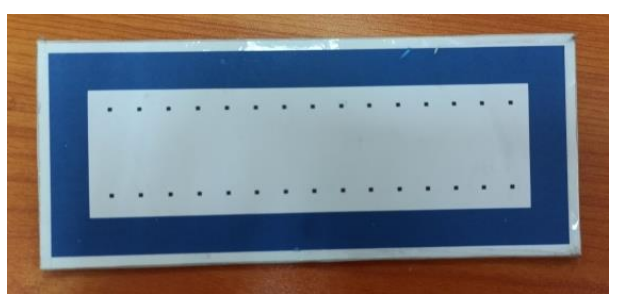

Figure 2. The proposed rectangle frame

As mentioned, the laser point is fixed and the frame moves with the body and we need to determine this motion to evaluate the imbalance in the Romberg test. If we consider the frame to be fixed, the motion of the laser point on the frame is the body's movement but in the opposite direction. The camera records a video of the frame, including the laser point on the frame, at 30-second intervals. Video is then processed to accurately determine the motion of the laser point on the frame. It is also the movement of the body.

\subsection{Key steps and algorithms}

The proposed processing algorithm is used to determine the movement of the reference laser point on the frame. To do this, the video is extracted into a series of images. Then the proposed blue frame area is extracted. Next, the background of the frame is extracted and distorted back to a rectangular shape (in case the camera moves in different positions during the rotation, causing the frame to no longer retain its rectangular shape). The proposed frame is surrounded by a blue outline to facilitate frame recognition if the patient is wearing a shirt of the same color as the background color of the frame. The laser point will be extracted from each rectangular frame. In each frame, virtual joins are connected between the two premark points of each pair. The position of the laser point to the first virtual ramp is calculated as the distance of the laser point to the position of the first left virtual join, plus the number of pairs of further left premark points. This calculation is illustrated in Figure 3.

\subsubsection{Tracking frame}

Figure 4 describes the proposed rectangular frame extraction algorithm. As mentioned, the advantage of this method is that the recording is flexible, which can use any type of camera (we use the camera available on Iphone phones, recording speed 30 frames per second), the camera does not required to be fixed, as long as the proposed rectangular frame is always recorded in the video for analysis. The video captures the movement of a rectangular frame mounted on the body, along with a red laser source projected onto the rectangular frame as a red dot. RGB color image sequence extracted from video. Then the rectangular frame image is separated from the image. To facilitate the separation of this rectangular frame, the outer border of the rectangular frame will be bounded by a blue border, which facilitates the correct separation of the frame in case the patient wears a shirt of the same color as the background of the proposed frame (here in white color). Rectangular frame with blue border, white background and black pairs' dots. If the brightness is changed during recording the video, the RGB image will give different R, G, and B color values, it will be difficult to detect the blue border. During movie shooting, the brightness of the environment may be changed, so RGB color images need to be converted to hue, saturation and value (HSV) space. The original RGB image as shown in Figure 5(a) is converted to an HSV image, creating a color threshold to extract the blue area. The blue contour of the rectangular frame will be extracted, the result shown in Figure 5(b). The determination of the laser point area is performed similarly to the blue frame extraction step, but according to the color range of the laser point area as shown in Figure 5(c). Then determine the center of this area and take this point as the coordinate of the laser point.

When creating color thresholds in HSV images, some other objects with the same color range as the blue border will also be extracted. Therefore, at this step, it is necessary to apply some conditions to accurately determine the proposed framework. The condition here is that the selected frame must have a quadrangular shape and the laser point must be in that extraction region. From the binary image after the blue filter as shown in Figure 5(b), contours are used to define the shape of the extracted region. The contour is the coordinates of the points along the boundary of the object. Select the object as a quadrilateral, with the coordinates of the four vertices of the quadrilateral being the top left, bottom left, top right, and bottom right points. The coordinates of the vertices of the quadrilateral are compared with the coordinates of the laser point. The proposed frame is the quadrilateral to satisfy the condition containing laser point. Figure 5(d) shows the extracted frame. 

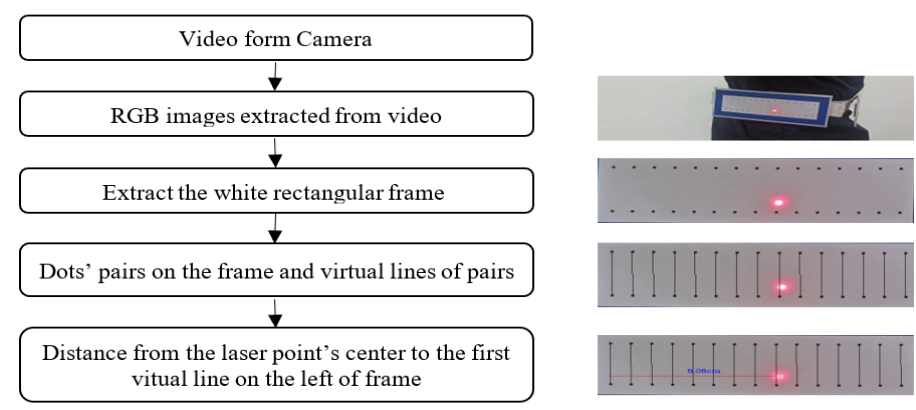

Figure 3. The flowchart of method

However, this frame can be distorted, not rectangular due to the camera angle. Therefore, this quadrangular frame needs to be re-calibrated into a rectangle according to the original scale. To increase accuracy, the blue border is removed, leaving only the white background inside. The same treatment as to find the background area. The four vertices of the background area are rescaled to create a rectangle as shown in Figure 5(e).
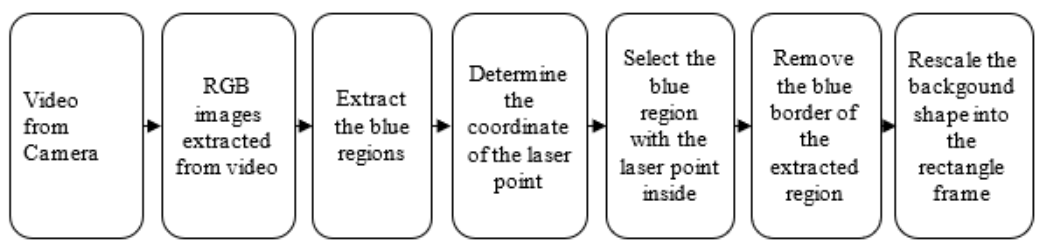

Figure 4. Flowchart of croping background frame algorithm

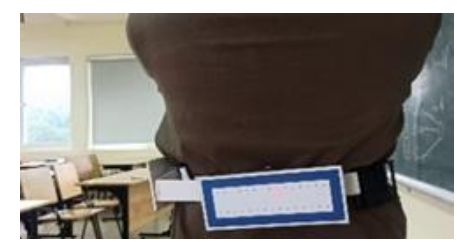

(a)

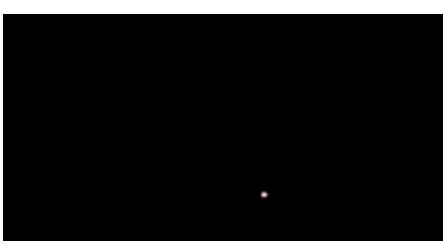

(c)

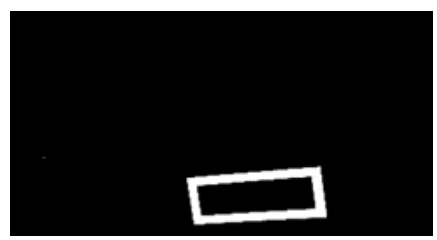

(b)

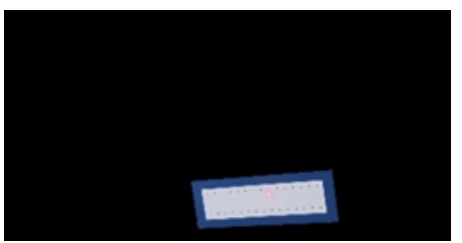

(d)

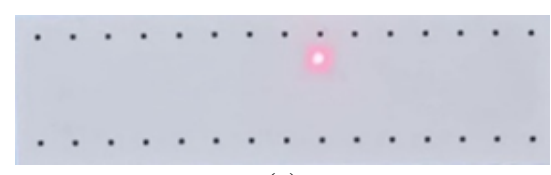

(e)

Figure 5. The tracking background frame steps: (a) image extracted from video, (b) mask of filtered blue area, (c) mask of extracted laser point, (d) the extracted area with laser point and black dots inside

\subsubsection{Movement determination}

The process of movement determination is given in Figure 6. As mentioned, in order to increase flexibility and reduce the complexity of the setup process, the proposed option allows users to move the camera during the recording process, as long as the proposed rectangular paper frame always recorded. In order to increase the accuracy in calculating the displacement of the laser point on the proposed background frame, this frame consists of 15 pairs of black dots, each $1 \mathrm{~cm}$ apart, which are considered as reference 
coordinates on the frame. The black dots and the center coordinates of these black dots will be extracted and calculated from the background frame image in Figure 5(e). The background image in Figure 5(e) is converted from an RGB image to a grayscale image and creates a histogram as shown in Figure 7(a) to serve to separate the black points according to their gray threshold on the histogram. Since lighting conditions can change during recording, which will affect the gray level value of the pixels in the image, the selection of the threshold to separate the black dots will be dynamically selected. Since the total number of pixels of the black dots is quite small compared to the total number of pixels of the extracted frame (about 100000 pixels), the gray threshold value is chosen under the condition that the total number of pixels has a gray level value from 0 to the value selected threshold is about 8000 pixels. The mask of black points is shown in Figure 7(b). After the center coordinates of the black points are determined, virtual lines connect between the central coordinates of the upper and lower pairs of black dots. These virtual lines are used as reference lines to calculate the distance of the laser position from the origin (first virtual line on the left), as shown in Figure 7(c).

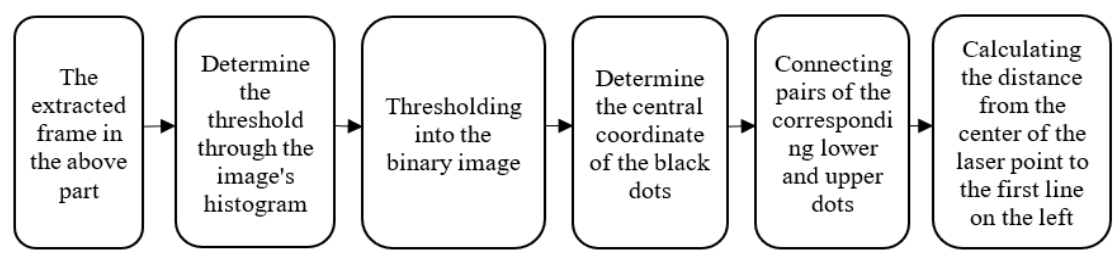

Figure 6. The flowchart of movement determination algorithm

This distance as shown in Figure 8, is calculated by counting the number of virtual lines from the second left line to the laser point $(\mathrm{N})$ and adding the distance from the center coordinate of the laser point to its nearest left virtual line $(\Delta \mathrm{d} / \Delta \mathrm{D})$. The result of measuring the distance from the laser point to the left first virtual line is shown in Figure 9. Since the laser point is fixed, the proposed background frame is attached to the object, so the relative motion of the laser point on the frameis also the motion of the object.

$$
\text { Distance }=\left(N+\frac{\Delta d}{\Delta D}\right) \times 1 \mathrm{~cm}
$$

Where $\mathrm{N}$ lines are also $\mathrm{N}$ centimeters because the distance between the lines is 1 centimeter.

$\Delta d$ : Number of pixel from center of laser point to its nearest left virtual line.

$\Delta \mathrm{D}$ : Total number of pixel between its nearest left and right virtual lines.

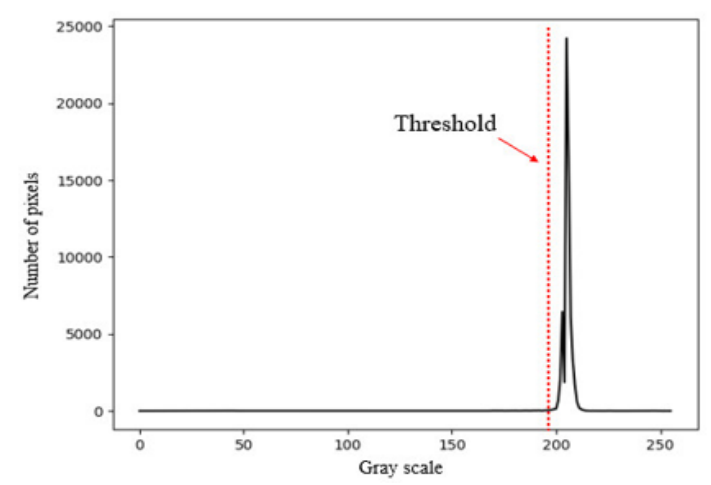

(a)

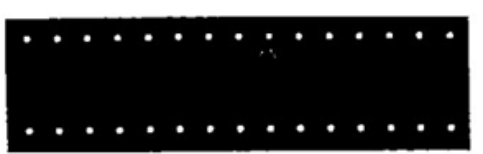

(b)

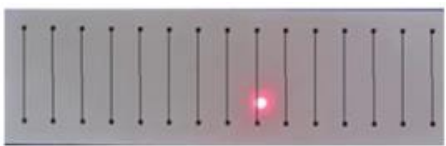

(c)

Figure 7. Determination of the position of the laser point on the proposed frame, (a) histogram of the detected background frame, (b) the binary image of background frame after thresholding step, and (c) virtual lines between corresponding upper and lower black dots 


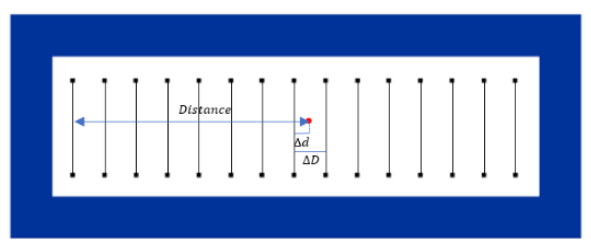

Figure 8. Laser point distance calculation

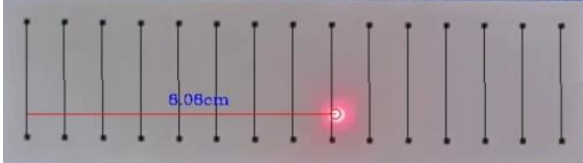

Figure 9. Distance of laser point to the first line on the left

\section{EXPERIMENTS}

\subsection{Examining accuracy}

Compared to the marker-less technique [45], [46], the marker-based technique has the advantage of high accuracy and stability in object tracking by identifying based on physical marker [47]. However, it is necessary to estimate the camera's pose (including position and rotation) in reality to refer to the obtained image [36], [46]. As a result, when camera moves from its initial position, it will cause disruption in the frame sequence [46]. In other words, the portability of the camera is limited.

Our proposed method can effectively solve the limitation. To prove this, we performed a test as shown in Figure 10. Firstly, the laser source is projected onto the frame and kept in a stationary state. Place the camera at the first position $\left(\alpha=90^{\circ}\right)$, then move the camera to the left in turn with distances (d) with the corresponding angles $(\alpha)$. The result is shown in Table 1 .

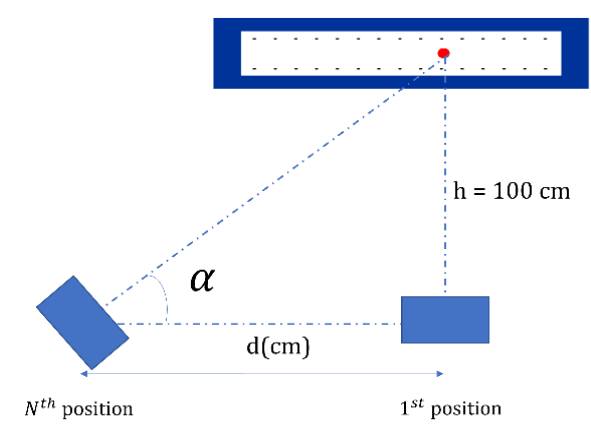

Figure 10. Experiment to test the flexibility of the proposed method

Table 1. Test results

\begin{tabular}{lccccccccc}
\hline \multicolumn{1}{c}{$\mathrm{d}(\mathrm{cm})$} & 0 & 20 & 40 & 60 & 80 & 100 & 120 & 140 & 160 \\
\hline Angle $(\alpha)$ & $90^{\circ}$ & $78.69^{\circ}$ & $68.20^{\circ}$ & $59.04^{\circ}$ & $51.34^{\circ}$ & $45^{\circ}$ & $39.81^{\circ}$ & $35.54^{\circ}$ & $32.01^{\circ}$ \\
Distance $(\mathrm{cm})$ & 4.92 & 4.92 & 4.92 & 4.92 & 4.92 & 4.92 & 4.90 & 4.87 & $\mathrm{X}$ \\
\hline
\end{tabular}

From Table 1, it can be seen clearly that except for the measument limit at $\mathrm{d}=160 \mathrm{~cm}$, the other positions give quite similar results. A difference of $0.05 \mathrm{~cm}$ per a range angle $\left(55^{\circ}\right.$ ) (from $\mathrm{d}=0$ to $140 \mathrm{~cm}$ ) is considered very small. This proves that our proposed method is not affected by the position of the camera during recording.

\subsection{Experimental setup}

As illustrated in Figure 2, the experiment was performed by using a portable camera, a red laser source, and a proposed rectangle frame. Height is the main factor influencing the body's sway [48]. Hence, in this study, the proposed frame was sticked to the volunteer's back, at around 0.53-0.56 (for female) and 0.530.59 (for male) of human's height. At this position, people have the strongest sway [32], [33]. During the test, the red laser source is anchored to the ground and projected onto the white region of the proposed frame. In this study, we use Iphone 7plus's camera to record the video for experiment, which has resolution of 1080x1920 pixel, and 30 frames per second.

The tests were carried out in a room with normal lighting, temperature, and sound, which had no negative impact on the patient's state during the procedure. The video was captured with the attached frame for 30 seconds after the objects started standing in tandem Romberg stance with closed eyes. The relative motions of the laser point in the frame are the patient's sway. 


\subsection{Experimental result and discussion}

The test was performed on a group of volunteers consisting of 35 healthy volunteers (no vertigo symptoms) and 3 patients with vestibular symptoms (headache, insomnia, dizziness), who were diagnosed by a doctor. Movement data of volunteers were collected and analyzed through 30-second videos. This data represents the position of the laser point relative to the origin. The data are normalized by converting the origin to the average value of the entire 30 seconds data segment.

The change in the position of the body over time is shown in Figure 11. It can be seen that the amplitude of the body position fluctuations is larger in vestibular patients than that in healthy volunteers. Each dataset for each volunteer contains $\mathrm{N}=900$ samples (30 samples x $30 \mathrm{fps}$ ) measured in time series.

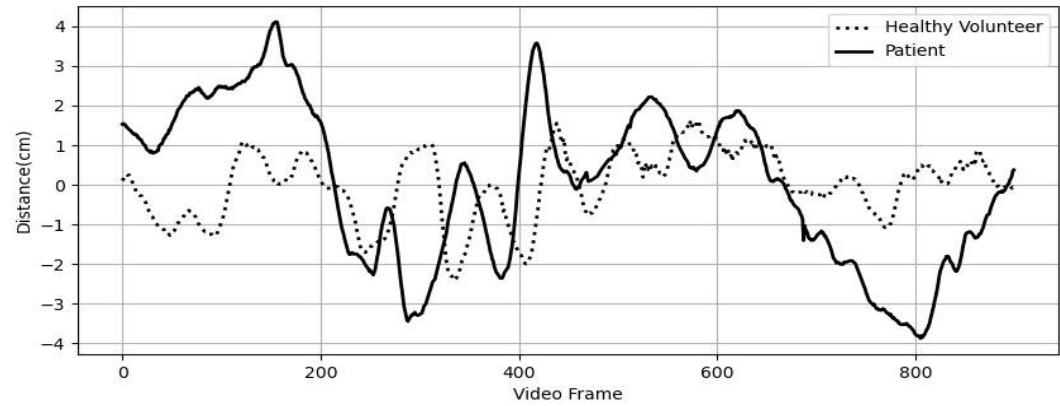

Figure 11. The position of the body's center of healthy volunteer and patient

According to study [34], [49]-[51], this data can be converted to more meaningful parameters: mean distance (MD), root mean square (RMS) distance. Figure 12 is a graph showing the difference in MD of healthy volunteers and patients. The average of MD values and its standard deviation (the black dashed lines) of healthy volunteers is $0.67 \mathrm{~cm} \pm 0.17 \mathrm{~cm}$, those values for patients is $1.7 \mathrm{~cm} \pm 0.1 \mathrm{~cm}$. This figure shows that the value of the mean distance of the group of patients is higher than that of the healthy volunteers group.

Figure 13 shows the difference in RMS distance of healthy volunteers and patients. The average of RMS values and its standard deviation (the black dashed lines) of healthy volunteers is $0.84 \mathrm{~cm} \pm 0.22 \mathrm{~cm}$, those values for patients is $2.04 \mathrm{~cm} \pm 0.11 \mathrm{~cm}$. This figure shows that the value of the RMS distance of the group of patients is higher than that of the healthy volunteers group. With the preliminary research resutls showed, patients with balance disorder and healthy people can be classified through the proposed method.

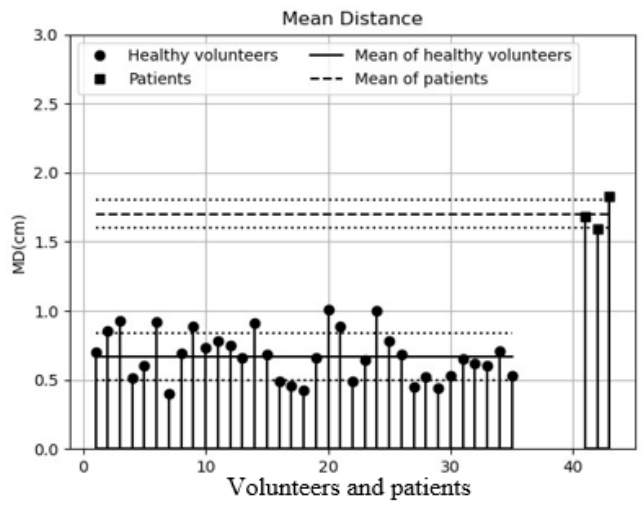

Figure 12. Mean distance

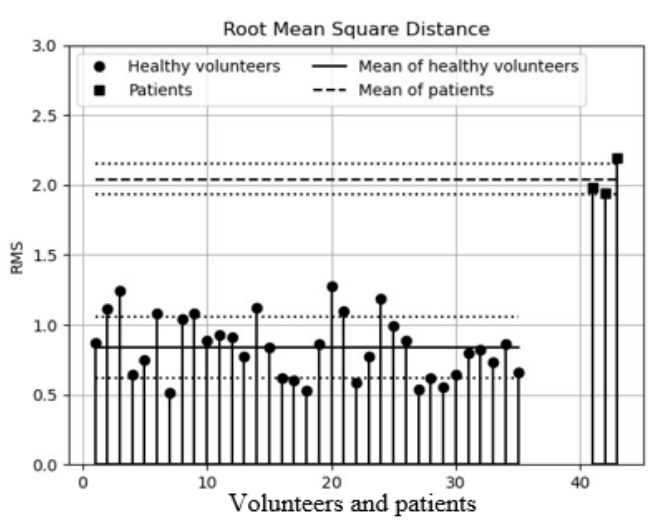

Figure 13. RMS distance

\section{CONCLUSION}

Our study demonstrated an image-processing-based technique for detecting and categorizing individuals with balance disorders. This method uses a simple, low-cost system, consisting of a red laser source, a proposed rectangular paper frame, a camera, and image processing software. The stationary laser source projects laser point onto the frame mounted on the patient's back. The camera may move as much as necessary in order to capture the frame properly. The black dots that are marked on the frame will reduce mistakes when the frame is slanted on viode or bent when mounted to the patient's back. The system was 
performed on vestibular patients and healthy people using a Romberg test. According to preliminary findings, the suggested method can correctly recognize human body movement during the Romberg test. It can also identify between two groups of people: sick and healthy people. As a result, this approach can be used to screen for vestibular diseases. The main advantages of this technology are its low cost, the flexibility of the measurement setting, and the fact that the person performing the test (videographer) does not require high medical expertise.

\section{REFERENCES}

[1] Y. Agrawal, B. K. Ward, and L. B. Minor, "Vestibular dysfunction: Prevalence, impact and need for targeted treatment," Journal of Vestibular Research, vol. 23, no. 3, pp. 113-117, 2013, doi: 10.3233/VES-130498.

[2] Y. Agrawal, J. P. Carey, C. C. D. Santina, M. C. Schubert, and L. B. Minor, "Disorders of balance and vestibular function in US adults: data from the National Health and Nutrition Examination Survey," Arch Intern Med., vol. 169, no. 10, pp. 938-944, 2009, doi: 10.1001/archinternmed.2009.66.

[3] S. Brosel and M. Strupp, "The Vestibular System and Ageing," Biochemistry and Cell Biology of Ageing: Part II Clinical Science. Subcellular Biochemistry, vol. 91, 2019, pp. 195-225, doi: 10.1007/978-981-13-3681-2_8.

[4] H. Neuhauser, "The epidemiology of dizziness and vertigo," Handb Clin Neurol., vol. 137, pp. 67-82, 2016, doi: 10.1016/B978-0444-63437-5.00005-4.

[5] T. L. Thompson and R. Amedee, "Vertigo: A Review of Common Peripheral and Central Vestibular Disorders," The Ochsner Journal, vol. 9, no. 1, pp. 20-26, 2009, PMCID: PMC3096243, PMID: 21603405.

[6] A. D. Stadio et al., "Loop characteristics and audio-vestibular symptoms or hemifacial spasm: is there a correlation? A multiplanar MRI study," European Radiology, vol. 30, pp. 99-109, 2020, doi: 10.1007/s00330-019-06309-2.

[7] Kelsey Hatton, "Diagnostic Tests for Vestibular Disorders," $2015 . \quad$ [Online]. Available: https://vestibular.org/sites/default/files/Diagnostic-Tests-for-Vestibular-Disorders_26.pdf

[8] H. S. Cohen, "A review on screening tests for vestibular disorders," Journal of Neurophysiology, vol. 122, no. 1, pp. 81-92, 2019, doi: 10.1152/jn.00819.2018.

[9] M. Strupp, J. Dlugaiczyk, B. B. Ertl-Wagner, D. Rujescu, M. Westhofen, and M. Dieterich, "Vestibular Disorder," Deutsches Arzteblatt international, vol. 117, no. 17, pp. 300-310, 2020, doi: 10.3238/arztebl.2020.0300.

[10] K. Campbell, “Essential Audiology for Physicians (Singular Audiology Text)," Cengage Learning, 1 st edition, 1997.

[11] D. L. McCaslin, “Electronystagmography and Videonystagmography: ENG/VNG," 2nd edition, Plural Publishing, Inc., 2019.

[12] N. Bhattacharyya et al., "Clinical Practice Guideline: Benign Paroxysmal Positional Vertigo (Update)," Otolaryngology-Head and Neck Surgery, vol. 156, no. 3S, pp. S1-S47, 2017, doi: 10.1177/0194599816689667.

[13] M. Ashiria, B. Lithgow, A. Suleiman, B. Mansouri, and Z. Moussavi, "Electrovestibulography (EVestG) application for measuring vestibular response to horizontal pursuit and saccadic eye movements," Biocybernetics and Biomedical Engineering, vol. 41, no. 2, pp. 527-539, 2021, doi: 10.1016/J.BBE.2021.03.007.

[14] J. Colebatch, "SY2.7. Evaluation of the vestibular system: cVEMP and oVEMP," Clinical Neurophysiology, vol. 132, no. 8, pp. e43, 2021, doi: 10.1016/j.clinph.2021.02.034.

[15] A. Patil and S. A. Sarda, "Usefulness of modified Romberg test in screening persons with vestibular dysfunction," Indian Journal of Otology, vol. 25, no. 2, pp. 66-70, 2019, doi: 10.4103/indianjotol.INDIANJOTOL_56_18.

[16] M. Ayasa and A. AlAmadib, "Emerging and distinct video head impulse test responses in elderly with vestibular symptoms," Braz. J. Otorhinolaryngology, 2021, doi: 10.1016/j.bjorl.2021.02.011.

[17] A. S. Schroeder et al., "General Movement Assessment from videos of computed 3D infant body models is equally effective compared to conventional RGB video rating," Early Human Development, vol. 144, 2020, doi: 10.1016/j.earlhumdev.2020.104967.

[18] S. S. M. Fong, S. S. M. Ng, L. M. Y. Chung, W. Y. Ki, L. P. Y. Chow, and D. J. Macfarlane, "Direction-specific impairment of stability limits and falls in children with developmental coordination disorder: Implications for rehabilitation," Gait Posture, vol. 43, pp. 60-64, 2016, doi: 10.1016/j.gaitpost.2015.10.026.

[19] R. H. Geuze, "Postural control in children with developmental coordination disorder," Neural Plast., vol. 12, no. 2-3, pp. 183-96, 2005, doi: 10.1155/NP.2005.183.

[20] J. J. Macnab, L. T. Miller, and H. J. Polatajko, "The search for subtypes of DCD: is cluster analysis the answer?," Hum Mov Sci., vol. 20, no. 1-2, pp. 49-72, 2001, doi: 10.1016/S0167-9457(01)00028-8.

[21] W. Aertssen, E. Bonney, G. Ferguson, and B. S.-Engelsman, "Subtyping children with developmental coordination disorder based on physical fitness outcomes," Hum Mov Sci., vol. 60, pp. 87-97, 2018, doi: 10.1016/j.humov.2018.05.012.

[22] S. Pieters, H. Roeyers, Y. Rosseel, H. V. Waelvelde, and A. Desoete, "Identifying subtypes among children with developmental coordination disorder and mathematical learning disabilities, using model-based clustering," J Learn Disabil ., vol. 48, no. 1, pp. 83-95, 2015, doi: 10.1177/0022219413491288.

[23] K. Asonitou and D. Koutsouki, "Cognitive process-based subtypes of developmental coordination disorder (DCD)," Hum Mov Sci., vol. 47, pp. 121-134, 2016, doi: 10.1016/j.humov.2016.01.002.

[24] N. Reicke, "The romberg head-shake test within the scope of equilibrium diagnosis," HNO, vol. 40, no. 6, pp. 195-201, 1997.

[25] F. O. Black, C. W. III, H. E. Rockette Jr., and R. Kitch, "Normal subject postural sway during the Romberg test," Am $J$ Otolaryngol, vol. 3, no. 5, pp. 309-318, 1982, doi: 10.1016/s0196-0709(82)80002-1.

[26] S. Kim, M. Kim, N. Kim, S. Kim, and G. Han, "Quantification and validity of modified romberg tests using three-axis accelerometers," Communications in Comp. and Information Sci., vol 338, pp. 254-261, doi: 10.1007/978-3-642-35251-5_35.

[27] J. A. Goebel, "Practical Management of the Dizzy Patient 2nd Edition," Lippincott Williams \& Wilkins, 2008.

[28] G. M. Halmágyi and I. S. Curthoys, "Vestibular contributions to the Romberg test: Testing semicircular canal and otolith function," European Journal of Neurology, vol. 28, no. 9, pp. 3211-3219, 2021, doi: 10.1111/ene.14942.

[29] J. Forbes and H. Cronovich, Romberg Test, StatPearls Publishing LLC, 2021.

[30] K. A. McMichael, J. V. Bilt, L. Lavery, E. Rodriguez, and M. Ganguli, "Simple Balance and Mobility Tests Can Assess Falls Risk When Cognition Is Impaired," Geriatr Nurs., vol. 29, no. 5, pp. 311-323, 2008, doi: 10.1016/j.gerinurse.2007.10.016.

[31] DE. O’Neill, KM. Gill-Body, and DE. Krebs, "Posturography changes do not predict functional performance changes," The American Journal of Otology, vol. 19, no. 6, pp. 797-803, 1998, PMID: 9831157. 
[32] T. A. Vu, H. Q. Huy, N. A. Tu, L. V. Tuan, L. V. Khanh, and P. T. V. Huong, "The models of Relationship Between Center of Gravity of Human and Weight, Height and 3 Body's Indicators (Chest, Waist and Hip)," Journal of Science \& Technology technical Universities, vol. 139, pp. 57-61, 2019.

[33] A. Bakhtiari, F. Bahrami, and B. N. Araabi, "Real time estimation and tracking of human body Center of Mass using 2D video imaging," 1st Middle East Conf. on Bio. Eng., 2011, pp. 138-141, doi: 10.1109/MECBME.2011.5752084.

[34] T. E. Prieto, J. B. Myklebust, R. G. Hoffmann, E. G. Lovett, and B. M. Myklebust, "Measures of postural steadiness: differences between healthy young and elderly adults," IEEE Trans Biomed Eng., vol. 43, no. 9, pp. 956-966, 1996, doi: 10.1109/10.532130.

[35] M. H. Khan, M. Zöller, M. S. Farid, and M. Grzegorzek, "Marker-Based Movement Analysis of Human Body Parts in Therapeutic Procedure," Sensors, 20, no. 11, pp. 3312, 2020, doi: 10.3390/s20113312.

[36] A. Katiyar, K. Kalra, and C. Garg, "Marker based augmented reality," Advances in Computer Science and Information Technology (ACSIT), vol. 2, no. 5, pp. 441-445, 2015.

[37] Y. Tao and H. Hu, "Colour based human motion tracking for home-based rehabilitation," 2004 IEEE International Conference on Systems, Man and Cybernetics, vol. 1, pp. 773-778, 2004, doi: 10.1109/ICSMC.2004.1398396.

[38] D. Wagner, T. Pintaric, F. Ledermann, and D. Schmalstieg, "Towards Massively Multi-user Augmented Reality on Handheld Devices," Lecture Notes in Computer Science, vol. 3468, pp. 208-219, 2005, doi: 10.1007/11428572_13.

[39] J. W. Burke, P. J. Morrow, M. D. J. McNeill, S. M. McDonough, and D. K. Charles, "Vision Based Games for Upper-Limb Stroke Rehabilitation," 2008 International Machine Vision and Image Processing Conference, 2008, pp. 159-164, doi: 10.1109/IMVIP.2008.16

[40] D. Rado, A. Sankaran, J. Plasek, D. Nuckley, and D. F. Keefe, “A real-time physical therapy visualization strategy to improve unsupervised patient rehabilitation," IEEE Trans. on Visualization and Computer Graphics, 2009.

[41] G. Paolini et al., "Validation of a method for real time foot position and orientation tracking with microsoft kinect technology for use in virtual reality and treadmill based gait training programs," IEEE Transactions on Neural Systems and Rehabilitation Eng., vol. 22, no. 5, pp. 997-1002, 2014, doi: 10.1109/TNSRE.2013.2282868.

[42] C. C. Chen, C. Y. Liu, S. H. Ciou, S. C. Chen, and Y. L. Chen, "Digitized Hand Skateboard Based on IR-Camera for Upper Limb Rehabilitation," Journal of Medical Systems, vol. 41, no. 2, p. 36, 2017, doi: 10.1007/s10916-016-0682-3.

[43] G. R. D. Bernardina, T. Monnet, H. T. Pinto, R. M. L. de Barros, P. Cerveri, and A. P. Silvatti, "Are Action Sport Cameras Accurate Enough for 3D Motion Analysis? A Comparison with a Commercial Motion Capture System," Journal of Applied Biomechanics, vol. 35, no. 1, pp. 80-86, 2019, doi: 10.1123/jab.2017-0101.

[44] J. Malus, J. Skypala, J. F. Silvernail, and J. Uchytil, "Marker Placement Reliability and Objectivity for Biomechanical Cohort Study: Healthy Aging in Industrial Environment (HAIE-Program 4)," Sensors, vol. 21, no. 5, p. 1830, 2021, doi: $10.3390 / \mathrm{s} 21051830$.

[45] S. L. Colyer, M. Evans, D. P. Cosker, and A. I. Salo, "A review of the evolution of vision-based motion analysis and the integration of advanced computer vision methods towards developing a markerless system," Sports Med - Open, vol. 4, no. 1, p. 24, 2018, doi: 10.1186/s40798-018-0139-y.

[46] A. S.-Niaraki and S. M. Choi, "A Survey of Marker-Less Tracking and Registration Techniques for Health and Environmental Applications to Augmented Reality and Ubiquitous Geospatial Information Systems," Sensors, vol. 20, no. 10, p. 2997, 2020, doi: $10.3390 / \mathrm{s} 20102997$

[47] D. Wagner, A. Mulloni, T. Langlotz, and D. Schmalstieg, "Real-Time Panoramic Mapping and Tracking on Mobile Phones," 2010 IEEE Virtual Reality Conference (VR), 2010, pp. 211-218, doi: 10.1109/VR.2010.5444786.

[48] H. Nakagawa, T. Yoshida, and K. Yamada, "Mechanical analysis of the influence of the change in the height of gravitational center on body sway properties for postural control in the human body," Recent Advances in Structural Integrity Analysis Proceedings of the International Congress, 2014, doi: 10.1533/9780081002254.305.

[49] J. A. Raymakers, M. M. Samson, and H. J. J. Verhaar, "The assessment of body sway and the choice of the stability parameter(s)," Gait \& Posture, vol. 21, no. 1, pp. 48-58, 2005, doi: 10.1016/j.gaitpost.2003.11.006.

[50] K. Fujio and Y. Takeuchi, "Discrimination of standing postures between young and elderly people based on center of pressure," Sci Rep, vol. 11, no. 1, p. 195, 2021, doi: 10.1038/s41598-020-80717-z.

[51] T. Prasertsakul, P. Kaimuk, W. Chinjenpradit, and W. Charoensuk, "Correlational study of the center of pressure measures of postural steadiness on five different standing tasks in overweight adults," Biomed Tech (Berl), vol. 65, no. 3, pp. 367-378, 2020, doi: $10.1515 / \mathrm{bmt}-2018-0115$

\section{BIOGRAPHIES OF AUTHORS}

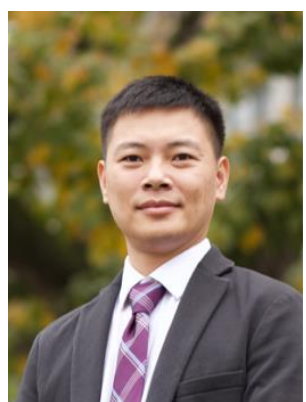

Dr. Tran-Anh Vu (iD S SC P is a senior lecturer and Vice Head of Biomedical Engineering Department, School of Electronics and Telecommunications, Hanoi University of Science and Technology, Hanoi, Vietnam. He earned his Ph.D. degree in Electrical Engineering from the University of Massachusetts at Lowell (USA) in 2014, MS degree in Biomedical Engineering from Tufts University (USA) in 2010, MS degree in Electronics and Telecommunication from Hanoi University of Science and Technology (Vietnam) in 2002, and BS degree in Electronics and Telecommunications from Hanoi University of Science and Technology (Vietnam) in 2000. His main research interests include the medical data analysis and classification, research and development applications for rehabilitation, smart health. He can be contacted at email: Vu.trananh@hust.edu.vn. 


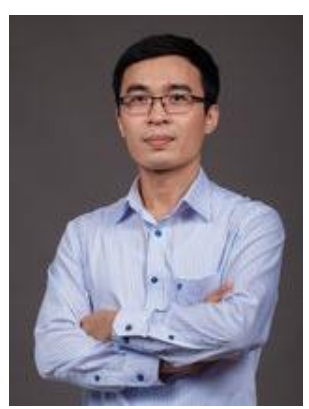

Hoang-Quang Huy (D) $\mathrm{SC}$ P is a lecturer of Biomedical Engineering Department, School of Electronics and Telecommunications, Hanoi University of Science and Technology, Hanoi, Vietnam. He earned his MS degree in Electronics and Telecommunication from Hanoi University of Science and Technology (Vietnam) in 2006, and BS degree in Electronics and Telecommunications from Hanoi University of Science and Technology (Vietnam) in 2002. His main research interests include the medical data analysis and classification, research and development applications for rehabilitation, smart health, hospital information systems. He can be contacted at email: Huy.hoangquang@mail.hust.edu.vn.

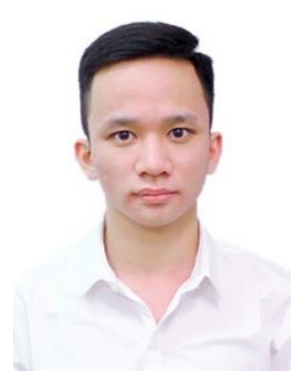

Le Van Tuan (D) SC SC Ptained his B.Sc in Biomedical Engineering Department, School of Electronics and Telecommunications, Hanoi University of Science and Technology, Hanoi, Vietnam in 2021. His main research interests include the medical data analysis and classification, research and development applications in Biomedical Engineering. He can be contacted at email: leanhtuan.311098@gmail.com.

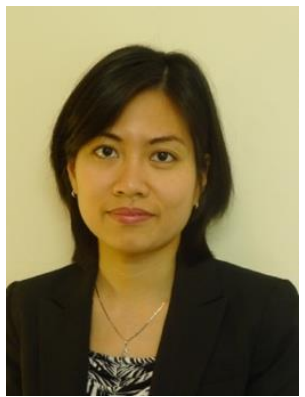

Dr. Pham-Thi-Viet Huong (D) Bd SC P obtained her B.Sc in Electrical Engineering from Hanoi University of Science and Technology in 2007. She got her MSc and PhD, both in Electrical Engineering, from University of Massachusetts Lowell in the United States, in 2010 and 2012. From 2012 to 2015, she was a researcher in the Manning School of Business, Lowell, Massachusetts. From 2017-2020, she was the faculty of VNU University of Engineering and Technology, Vietnam (VNU-UET). Since 2020, she works in International School-VNU. She is interested in data mining and analytics, machine learning methodologies, with applications in Biomedical Engineering. She can be contacted at email: Huongptv@isvnu.vn. 